Retroperitoneale Lymphdrüsen ziemlich stark vergrößert.

Erst die Kopfsektion ergab ein positives Resultat. Es fand sich: ein haselnuBgroBer, derber Tumor der Hypophyse; eine geschwulstartige Infiltration des Infundibulums und ein kirschkerngrober Tumor der Epiphyse. Es wurde nun der Verdacht einer pluriglandulären Erkrankung der endokrinen Drüsen ausgesprochen und Schilddrüse, Parathyreoidea, Nebennieren, Pankreas, Thymus und Ovarien nebst Stückchen der andern Organe zur histologischen Untersuchung eingelegt. Die-histologische Untersuchung des Hypo- und Epiphysentumors sowie des infiltrierten Infundibulums ergab überraschenderweise einen tuberkulösen Prozeß. - Zahlreiche, meist verkäste Konglomerattuberkel mit vielen Riesenzellen durchsetzen den Vorder- und Hinterlappen der Hypophyse und das Infundibulum. An drüsigen Elementen sind fast nur neutrophile Zellen zu sehen. Auch an der Neurohypophyse sind nervöse Elemente zu erkennen. Eine intermediäre Zone kann nicht nachgewiesen werden, doch wurden wegen Schonung des Präparates nur einige Scheibchen geschnitten. Das Gewebe der Epiphyse ist bis auf einige Kalkkonkremente vollständig durch den tuberkulösen Prozeß substituiert.

Sonst ergab die histologische Untersuchung der endokrinen Drüsen sowie der andern Organe bis auf eine Tuberkulose der retroperitonealen Drüsen kein abnormes Resultat. Es mußte demnach der Gedanke der pluriglandulären Erkrankung, für welche auch nichts in der Anamnese, im Habitus der Leiche sprach, fallen gelassen werden. Und jetzt nach Kenntnis der Simmondsschen Beobachtungen, möchte ich nicht zweifeln, daß der Fall in diese Gruppe der hypophysären Kachexie gehört.

Er ist aber nur dadurch als solcher zu erkennen, weil die sonst gefundenen tuberkulösen Veränderungen durchaus nicht genügend waren, um die allmählich zum Tode führende Erschöpfung zu erklären. Wären schwerere tuberkulöse Prozesse in der Leiche gefunden worden, so hätte die Kachexie kaum auf die Tuberkulose der Hypophyse, die nach Simmonds (1. c.) und eigener Erfahrung nicht gar so selten ist, im Sinne einer Störung der Innensekretion bezogen werden können. Die Tuberkulose überhaupt hätte genügt.

Ist aber einmal die Kenntnis der hypophysären Kachexie geläufig, dann wird vielleicht öfter zur Klärung der klinisch erhobenen Schwächezustände, welche durch die gefundenen anatomischen Prozesse nicht völlig verständlich sind, z. B: an eine Tuberkulose der Hypophyse rekurriert werden können.

Es scheint mir ähnlich zu liegen wie beim Addison. Nur eine primäre Atrophie oder eine isolierte Tuberkulose der Nebennieren wirkt überzeugend, daß nur durch den Ausfall der Funktion dieser Drüse der Symptomenkomplex erzeugt worden ist.

Die Kombination der Hypophysen- und Epiphysentuberkulose in unserem Falle scheint mir vorläıfig nur als bemerkenswertes Kuriosum erwähnt werden zu können.

\title{
Zur Sektion der Nasenhöhle und ihrer Nebenhöhlen.
}

(Aus dem Pathologiseh-Anatomischen Institut der Deutschen Universität in Prag.)

\author{
Von
}

Prof. A. Ghon.

(Hierzu 5 Textfiguren.)

Wenn wir absehen von dem Verfahren, das v. Luschka angegeben hat und wonach ein Einblick in die Nasenrachenorgane dadurch erzielt wird, da $\beta$ der harte Gaumen nebst dem angrenzenden Teil der Nasenscheidenwand mit dem Stemmeisen von unten her nach Exartikulation des Unter- 
kiefers entfernt wird, und absehen von der Trephine von Klebs, die ein Stück der Schädelbasis heraussägt, um die Pharynx- und Nasenhöhle zugänglich zu machen, so war es die Methode von Schalle, die zuerst die Möglichkeit schuf, die Nasen- und Rachenorgane genau zu untersuchen. Aber der Umstand, daß Schalle dabei ein besonderes Gewicht auf die gleichzeitige Herausnahme der Gehörorgane legte, machte die Methode etwas unständlich und erforderte eigene Instrumente, so da $B$ sie sich nicht recht einbürgerte und heute kaum irgendwo geübt werden dürfte. Mit einer Bogensäge werden nach dieser Methode die Felsenbeinpyramiden mit dem hinteren Teil der Nase durch einen Sägeschnitt herausgenommen, der, von oben her gesehen, fast die Form eines Kleeblattes hat. Wurde der Schnitt richtig geführt, so umfaßt der ausgesägte Teil der Schädelbasis die Nasen-Rachen- und Gehörorgane im Zusammenhange nebst der Gaumenmuskulatur und allen Nerven und Gefäßen darin. Erst nach der Herausnahme des Präparates erfolgt seine Zerlegung.

Für die Fälle, wo es vor allem auf die Besichtigung der Nasenhöhle und ihrer Nebenhöhlen ankam, empfahl dann Harke den Sagittalschnitt durch die Mitte der Schädelbasis. Die Kopfhaut wird dabei, nachdem das Gehirn in gewöhnlicher Weise Weise herausgenommen wurde, vorn bis zu den Augenbogen und Nasenbeinen, hinten bis zu dem Hinterhauptloch abpräpariert, worauf ein Sagittalschnitt etwas rechts oder links von der Medianebene die Schädelbasis vom Hinterhauptloch bis zu den Nasenbeinen durchsägt. Die beiden Schädelhälften werden nun auseinandergebogen, nicht durchschnittene Schleimhautüberzüge des Nasen- und Rachendaches durchtrennt, die noch bestehenden Knochenverbindungen gebrochen und die beiden Schädellbälften aufgeklappt. Ein Schnitt mit einer Stichsäge durchsägt sodann senkrecht zum Sagittalschnitt die Schädelbasis beiderseits, dort wo die obere Wand des Nasen-Rachenraumes in die hintere übergeht, bis zur Seitenwand des Nasenrachenraumes, worauf ein zweiter Schnitt von dem Punkte, wo der Sagittalschnitt den Türkensattel durchschneidet, nach dem Endpunkte des ersten Schnittes jederseits ein rechtwinkliges Dreieck heraussägt. Der Sagittalschnitt hat die Keilbeinhöhle der Seite eröffnet, wo der Schnitt geführt wurde, während die andere Keilbeinhöhle erst durch den Stichsägeschnitt vom Türkensattel zur Rachenwand eröffnet wird. Durch $\mathrm{Ab}$ tragung der Nasenseheidewand wird auch die Nasenhöhle der andern Seite zugänglich, durch Abtragung der Muscheln und Resektion der Nasenseitenwand die Oberkieferhöhle jeder Seite. Stirnhöhlen und Siebbeinzellen können, soweit sie nicht schon eröffnet sind, nunmehr leicht aufgemacht werden.

An die Methode von v. Luschka lehnt sich die von Beneke an: Nach einem Schnitte vom Processus mastoideus zum Manubrium sterni beiderseits wird der dadurch geschaffene Hautlappen bis zum Rande des Unterkiefers präpariert und der Zungenmuskel gelöst, worauf weicher Gaumen, Kehlkopf und Ösophagus in gewöhnlicher Weise entfernt werden. Nach Entfernung der Parotisdrüsen wird sodann der Unterkiefer exartikuliert, herabgezogen und mit dem Hautlappen und der Unterlippe so nach oben gehoben, daß die Zähne auf dem Nasenrücken stehen. Mit dem Meißel werden hernach der Processus palatinus maxillae sup. und die Lamina horizontalis ossis palati dicht am Processus alveolaris derart durchsprengt, daß sie sich von der geschlossenen unversehrten Schleimhaut des Bodens der Nasenhöhle abheben lassen. Nach Inzision der Schleimhaut von der Choane aus liegt die Nase mit den Muscheln vor, die mit dem Septum abgetragen werden können, wodurch der Fingang zu den Highmoreshöhlen frei wird. Es ist dann leicht, die Siebbeinzellen und vom Rachen her die Keilbeinhöhlen zu präparieren, ebenso das Felsenbein mit der Tuba Eustachii herauszunehmen.

Ein vorzüglicher Einblick in die Highmoreshöhlen, den unteren Absehnitt der Nasenhöhle und den Epipharynx wird nach Löwe dadurch gewonnen, daß die Übergangsfalte der Oberlippenschleimhaut von einer Tuberositas maxillae superioris zur andern durchtrennt und die Weichteile des Gesichtes von der Fossa canina abgehoben werden, worauf der harte Gaumen vom Oberkieferkörper in horizontaler Richtung mit einem breiten Meißel getrennt und die Gaumenplatte nach unten geklappt wird. Dann werden die untere und mittlere Muschel und die Innenwand der 
Kieferhöhle abgetragen, so daß nun die Siebbeinzellen von unten her eröffnet werden können. Nach Entfernung der oberen Muschel und des Septums nebst Ausräumung der Nasenseiten wird die Basis cranii zugänglich.

Unter den bisher angeführten Methoden hält Chiari die von Harke angegebene für die beste, weil sie nicht nur eine vollständige Untersuchung der Nase und ihrer Nebenhöhlen ermöglicht, sondern sich auch für die Gewinnung von Impimaterial zu bakteriologisehen Zwecken vor-

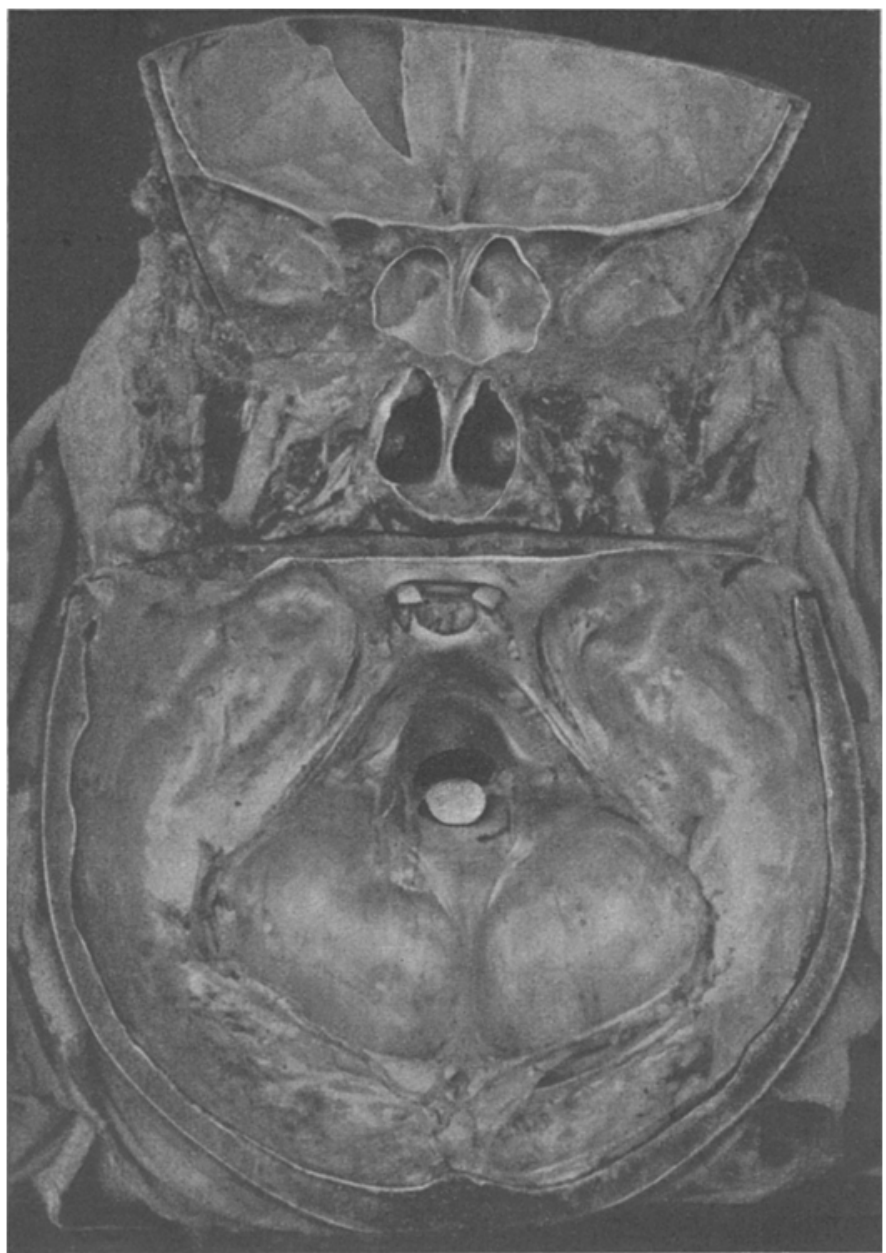

Fig. 1.

zïglich eignet. Thr gegenüber ist die Methode von Schall nach Chiari komplizierter und bringt nicht alle Teile der Nase genügend zur Anschauung. Auch die Methode ron Beneke steht der von Harke darin nach, daß die Keilbeinhöhlen und Siebbeinzellen dabei weniger gut zur Anschaaung gebracht werden. Und die Methode von Löwe hält Chiari deshalb nieht für unbedenklich, weil sie eine Verunstaltung des Gesichtes nicht ausschließt. 
Der Methade von Beneke nähert sich die von Westenhöffer, die die Nasenhöhle und Nasenmuscheln, das Ostium pharyngeum tubae und die Rachentonsille von unten her dadurch zur Anschauung bringt, daß nach Herausnahme der Halsorgane und vollständiger Freilegung des Unterkiefers, der nicht luxiert werden darf, bei herabhängendem Kopf der Leiche das Gaumendach vom Oberkiefer abgemeißelt wird. Ist die Sektion der Schädelhöhle nicht erlaubt, so entnimmt Westenhöffer die Knochen des Schädelgrundes mit den Nebenhöhlen der Nase in der Weise, daß er den Meißel mitten durch das Os basilare in die Schädelhöhle treibt und von dieser Öffnung aus das Keilbein mit der Stichsäge heraussägt, wobei er sich am Alveolarfortsatz des Oberkiefers hält.

Einen neuen Gesichtspunkt in der Frage brachte v. Hansemann mit seiner Luxationsmethode. Um den hinteren oberen Rachenraum mit der hinteren Fläche des weichen Gaumens und die Choane gut zur Anschauung zu bringen, luxiert $v$. Hansemann den Schädel nach vorn. Dazu wird die Haut des Schädels in der gewöhnlichen Weise zurückgeklappt, nur etwas weiter nach dem Nacken zu abpräpariert. Nachdem sodann alle Weichteile des Halses vom Hinterhaupt losgetrennt sind, werden die Gelenkflächen zwischen Hinterkopf und Atlas eröffnet und das Rückenmark durchschnitten. Ein noch besserer Einblick wird dadurch gewonnen, daß 2-3 Halswirbel herausgenommen werden.

Eine recht brauchbare Erweiterung erfuhr die Methode v. Hansemanns durch Oberndorfer. Nachdem der Schädel mit oder ohne vorherige Abnahme der Kalotte in der von y. Hansemann angegebenen Weise durch Lösung des Atlantookzipitalgelenkes nach vorn luxiert wurde, wird der Musculus temporalis abpräpariert, der äußere Gehörgang hart am Os temporale durchschnitten und das Unterkiefergelenk ausgelöst. Hernach werden die Bulbi nach Ablösung des Orbitalfettgewebes von dem Orbitaldach und nach Durchtrennung der Nervi optici nach vorn luxiert und dann die vordere Partie der Schädelbasis $1 \mathrm{~cm}$ oberhalb des oberen Orbitalrandes mit einer nicht zu breiten elastischen Stichsäge in horizontaler, stark nach hinten geneigter Richtung abgesägt. Das Dach der Orbita, die Lamina cribrosa und Crista galli. bleiben an der Schädelbasis. Zur vollständigen Lösung der Schädelbasis werden schlieBlich die Seitenwand der Orbita, das Siebbein, die Processus pterygoidei des Keilbeins und der Processus zygomaticus durchtrennt.

Nauwerck hingegen seziert die Nase und ihre Nebenhöhlen für gewöhnlich so, daß Keilbeinhöhlen, Siebbeinzellen, Stirnhöhlen und Highmoreshöhlen mit Hammer und MeiBel eröffnet und erst dann genauer untersucht werden, wenn sich die Notwendigkeit dazu herausstellt. Zur Eröffnung der Keilbeinhöhlen wird zunächst vor dem Türkensattel ein Meißelschlag frontal in das Keilbein geführt, dann der Meibel an der rechten Seite der Sella turcica möglichst wagrecht angesetzt und quer nach links durch die Keilbeinhöhlen getrieben und der Handgriff des Meißels hochgehoben, wodurch die Kuppe der Keilbeinhöhlen deckelartig abgehoben wird. Die Siebbeinzellen werden mit einem Krummeißel seitlich von der Crista galli zwischen Lamina cribrosa und Lamina papyracea eröffnet und die Stirnhöhlen mit Hohlmeißel und Hammer im vorderen und medialen Teíl der vorderen Schädelgrube, wenn sie nicht schon durch den Sägeschnitt des Sehädels eröffnet wurden. Um die Highmoreshöhlen von oben her darzustellen, wird das Orbitaldach und die híntere Hälfte des Bulbus entfernt, der Boden der Augenhöhle vom Foramen opticum bis nahe zum unteren Augenhöhlenrand von den Weichteilen befreit, ein Teil des großen Keilbeinflügels abgemeißelt, der abpräparierte Orbitalinhalt mit der Pinzette nach oben und vorn gezogen, der freigelegte Boden der Orbita ungefähr in der Größe eines Markstückes weggemeißelt und schließlich die Schleimhaut der Höhle eingeschnitten.

Nach Hauser wieder wird der Nasenrachenraum am einfachsten durch einen frontalen Sägeschnitt der Schädelbasis freigelegt. Dazu wird zunächst der Schnitt dureh die Kopfhaut. beiderseits nach unten erweitert, der äußere Gehörgang durchschnitten und das Ohr mit der Haut vom Schädel tief herabgelöst. Hernach wird durch die Mitte der Sella turcica ein frontaler Schnitt. 
etwas schräg nach hinten geführt, der die Schädelbasis in zwei Hälften trennt und das Unterkiefergelenk eröffnet. Nach Durchtrennung der Weichteile des Rachens können die beiden Hälften soweit auseinandergedrängt werden, daß die Nasenhöhle und ihre Nebenhöhlen vollständig über-

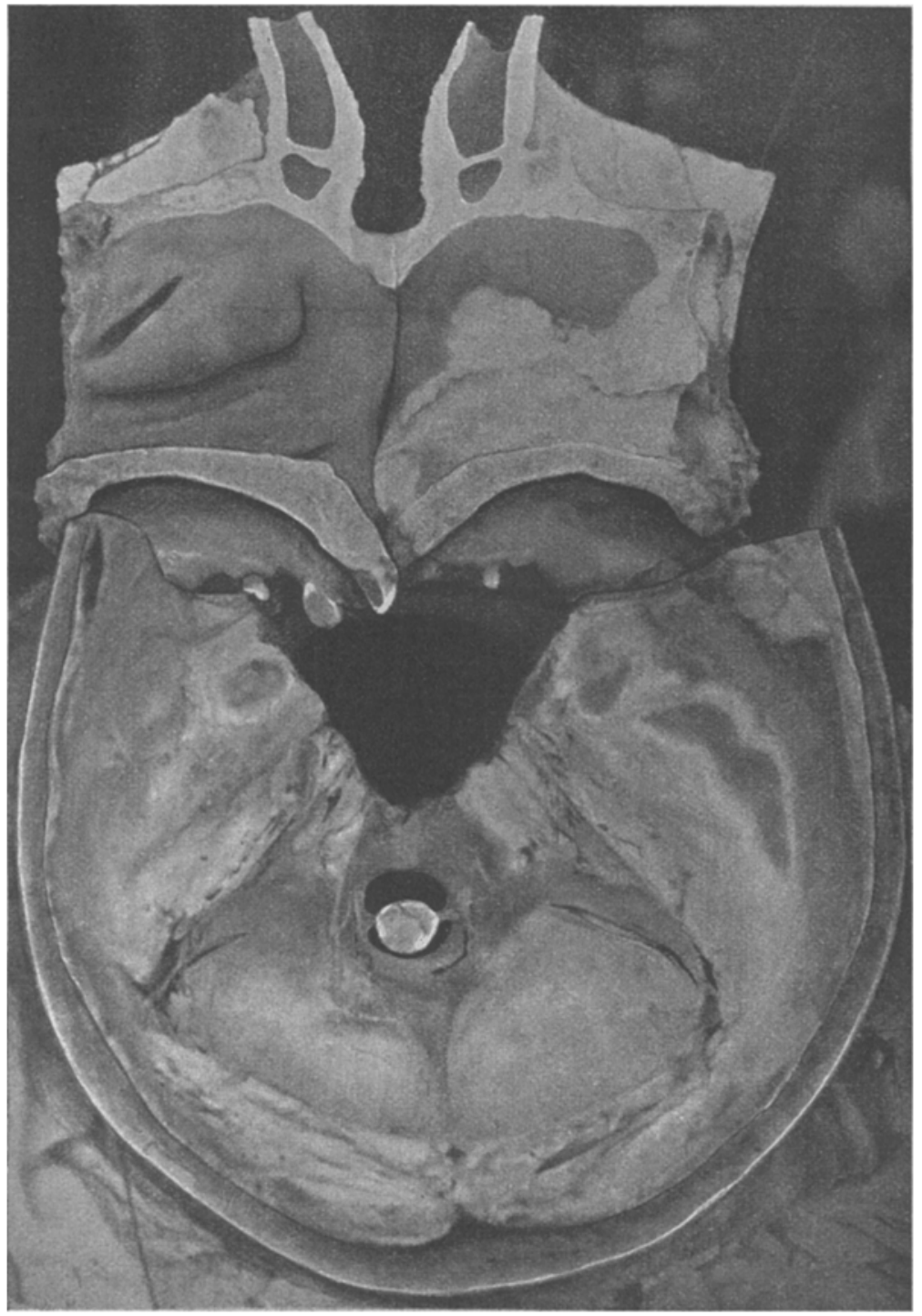

Fig. 2.

blickt werden. Meistens werden dabei anch die Keilbeinhöhlen schon eröffnet. Ist dies nicht der Fall, so können sie durch Abmeißelung ihrer hinteren Wand freigelegt werden. Die Eröffnung der Highmoreshöhlen erfolgt am besten schon vor dem frontalen Sägeschnitt vom Boden der eröffineten Orbitae, ähnlich wie es Nauwerck beschrieben hat. 
Loeschke endlich gebraucht den schon von Rokitansky geübten Frontalschnitt durch den Boden der vorderen Schädelgrube in ihrem hinteren Drittel, der nach Entz auch in Ungarn üblich ist und dort von Genersich eingeführt wurde. Dabei ist es notwendig, die Weichteile

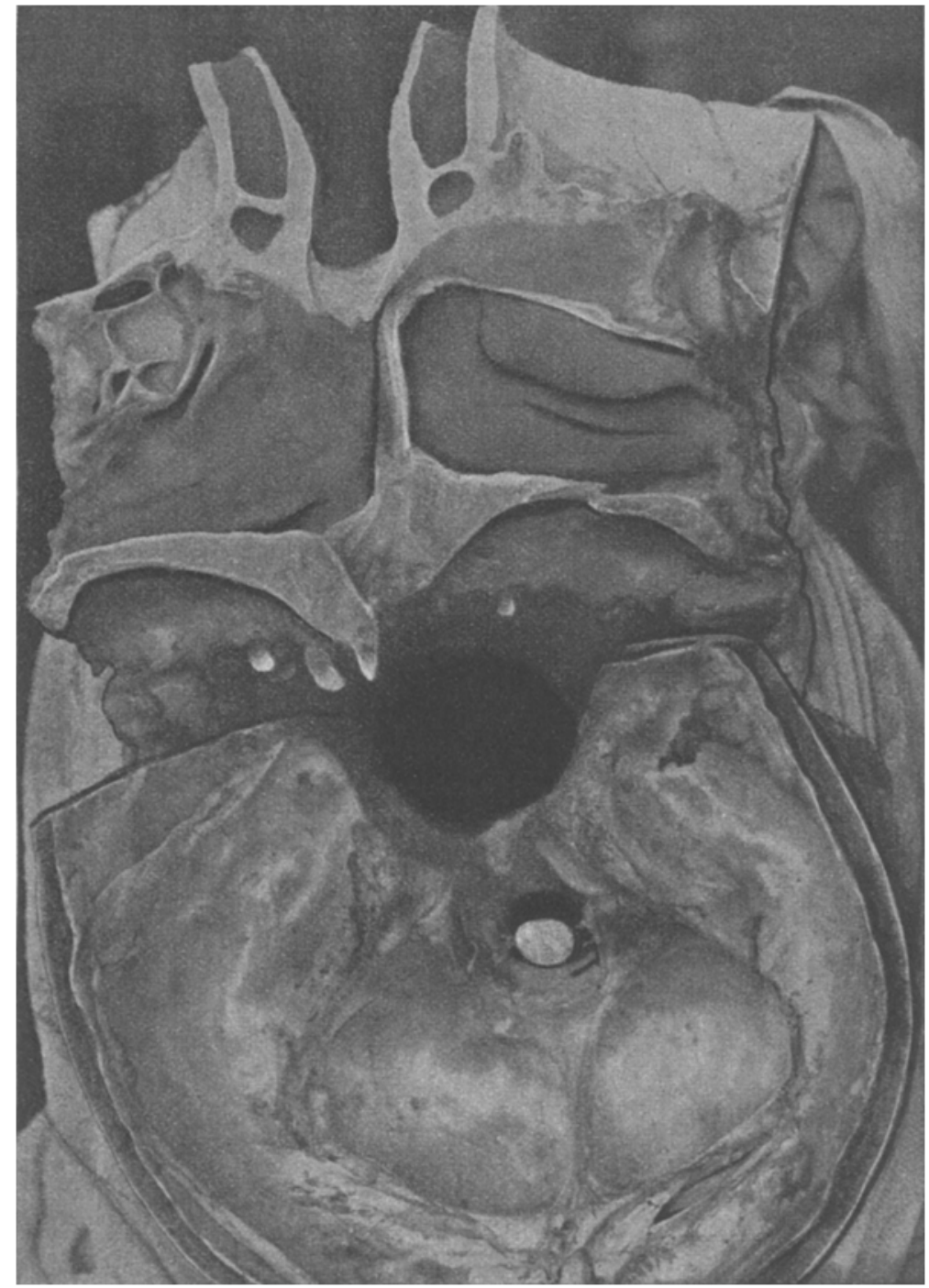

Fig. 3,

nach Durchtrennung des äußeren Gehörganges hart am Schädel so weit zurückzupräparieren, daß das Jochbein mit dem Jochbogen vollständig freiliegt. Der Schnitt wird so tief geführt, daß das Gaumendach und die Zahnreihe des Oberkiefers durehtrennt werden, wonach sich die beiden Schädelhälften anstandslos auseinanderklappen lassen und Nase, Siebbeinzellen und Highmores- 
höhlen im Querschnitte zur Darstellung gebracht werden. Die Keilbeinhöhlen werden dadurch. eröffnet, daß der Boden der Sella turcica durch einen horizontalen Meißelschlag abgehoben wird.

Der Methoden für die Sektion der Nasenhöhle und ihrer Nebenhöhlen gibt es sonach genug. Ein Teil der Methoden eröffnet die Naseñhöhle und ihre Nebenhöhlen oder wenigstens einen Teil

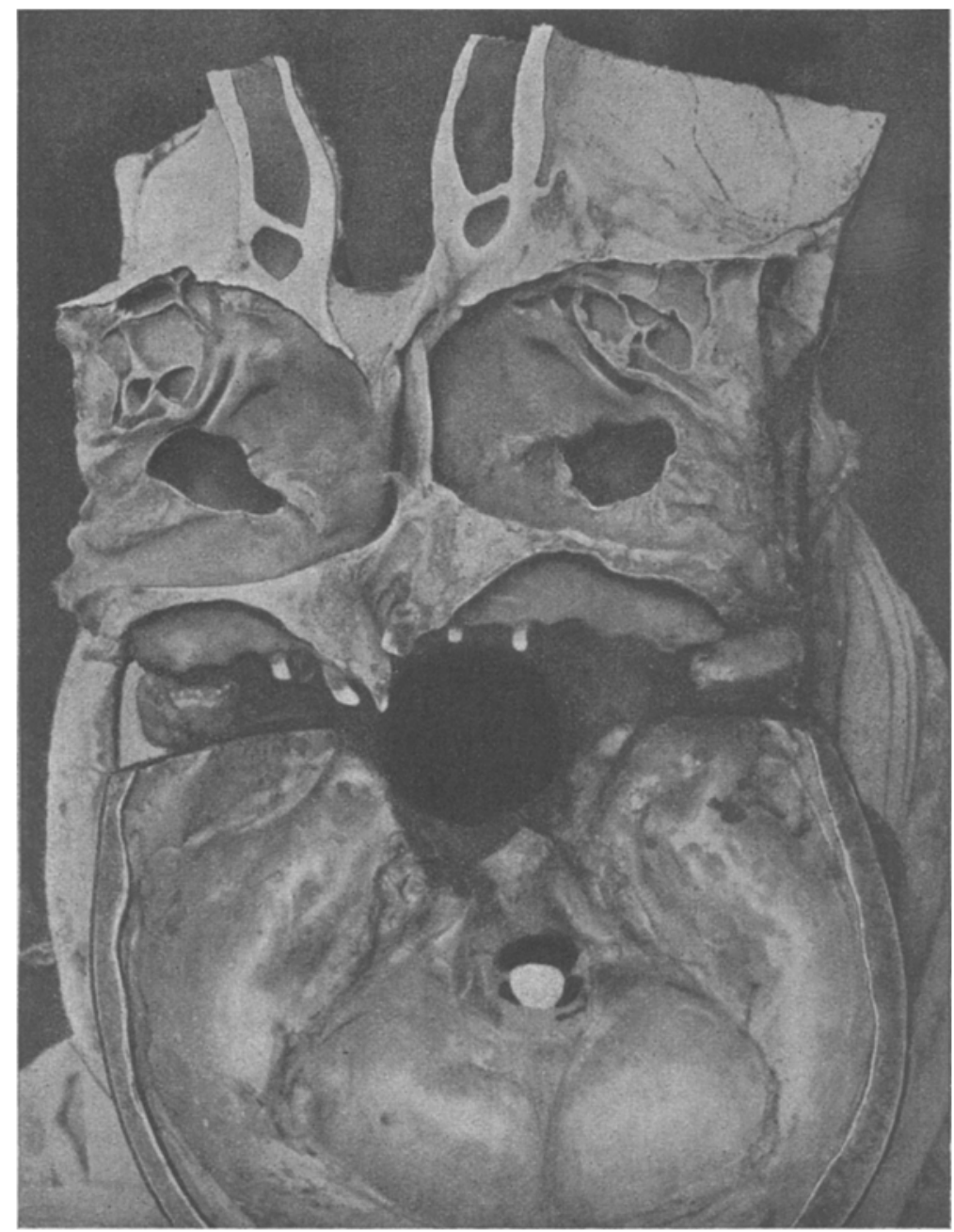

Fig. 4.

davon von unten her, also von der Mund-Rachenhöhle aus; ein anderer Teil von oben her, von der Schädelhöhle aus, entweder nach einem Sagittalschnitt durch die Mitte der Schädelbasis oder nach einem Frontalschnitt durch die schädelbasis, der in der vorderen oder in der mittleren Schädelgrube gefưhrt wird, oder schlieblich dadurch, daß die verschiedenen Höhlen einzeln aufgemacht werden; und ein dritter 'Teil nach Luxation des Schädels nach vorn.

Es wäre nach meiner Ansicht nicht richtig, wenn unter allen Umständen nur an einer einzigen der Methoden festgehalten würde. Sicher hat jede der Methoden ihre Vorteile, aber auch ihre. 
Nachteile. Allerdings scheint es, daß die Methoden, die die Nasenhöhle und ihre Nebenhöhlen von unten her eröffnen, im allgemeinen weniger im Gebrauche stehen als die, die von oben her eröffnen. Neben äußeren Umständen, die im einzelnen Falle in erster Linie füx die Wahl der Methode bestimmend sein werden, wird wohl der jeweilige Zweck, der mit der Eröffnung der Nasenhöhle und ihrer Nebenhöhlen veriolgt wird, dafür ausschlaggebend sein, welche von den Methoden herangezogen wird.

Untersuchungen über die Atiologie und Genese der eitrigen Meningitis, die ich im Institut von Hofrat Weichselbaum begonnen und in Prag weitergeführt habe, gaben mir Veranlassung, in allen untersuchten Fällen von Meningitis die Nasenböhle und ihre Nebenhöhlen anatomisch und bakteriologisch zu untersuchen. Dabei waren es nicht so sehr die anatomischen als vielmehr die bakteriologischen Ergebnisse der Untersuchungen, die mich dazu führten, mir eine meines Wissens bei uns nicht geübte Methode zurechtzulegen, die ich hier kurz beschreiben möchte. Sie gab mir befriedigendere Resultate als die bis dahin von mir benutzte Methode von Rokitansky und die von Harke und brauchte anch nach der Mitteilung der Methode von Oberndorfer nicht wieder aufgegeben zu werden.

Die Methode vereinigt den sagittalen und frontalen Schnitt durch die Schädelbasis von oben her und verbindet damit die besonderen Vorteile beider Schnitte. In ähnlicher Weise wie für die Methoden von Harke und Rokitansky wird der frontale Schnitt durch die Kopfschwarte beiderseits möglichst tief nach unten geführt, dabei der äußere Gehörgang durchschnitten, die hintere Hälfte der Kopfschwarte in gewöhnlicher Weise abgezogen, die vordere hingegen möglichst weit über das Gesicht herunter, nachdem sie vorn bis zur Nasenwurzel und zu den Supraorbitalrändern, seitlich bis zu den Jochbogen abgelöst wurde. Nachher wird die Kalotte des Schädeldaches in gewöhnlicher Weise durchschnitten und abgehoben und nun zunächst der frontale Schnitt durch die Sehädelbasis geführt, knapp vor der Sella turciea, so daß die Sella mit der Hypophyse der hinteren Hälfte des Schädels zufällt, Der Schnitt hält sich also ungefähr in der Witte zwischen dem Schnitt von Rokitansky und dem von 'Hauser und endet beiderseits, indem er etwas schief nach hinten und unten zieht, vor dem Unterkiefergelenk, das eröffnet und gelöst wird. Mit der Sella turcica und Hypophyse bleibt auch der Nasenrachenraum bei der hinteren Schädelhälfte, kann nach Durchtrenmung der Weichteile vollkommen überblickt werden und ist dadurch jedem Eingriff für bakteriologische und histologische Zwecke zugänglich. Die Keilbeínhöhlen werden durch den Schnitt eröffnet (Textfig. 1). Soll die Sella turcica mit der Hypophyse und der Nasenrachenraum mit der Rachentonsille und den Tubenostien besonders verarbeitet werden, so können diese Teile aus der hinteren Schädelhälfte in Form eines dreieckigen Stïckes herausgesägt werden. Die dazu notwendigen zwei Sägeschnitte werden seitlich von der Sella konvergierend gegen das Foramen magnum geführt (Textfig. 2-4).

Der Vorteil dieses Schnittes besteht nach meiner Ansicht vor allem darin, daß es dadurch möglich ist, die Sella tureica mit der Hypophyse und dem Nasenrachenraum im Zusammenhang zu gewinnen. Ich habe damit instruktive Präparate der Rachentonsille bei Kindertuberkulose und bei der Meningitis Weichselbaum gewonnen. Wird kein Wert auf den Zusammenhang der Hypophyse mit dem Rachendach gelegt, so kann der frontale Schnitt auch etwas weiter hinten geführt werden.

Der sagittale Schnitt, der nun folgt, wird zum Unterschied von der Methode Harkes nur durch die vordere Hälfte der Schädelbasis geführt. Er kann wie bei Harke etwas seitlich der Medianebene gehen oder genau in der Mitte. Tch ziehe im allgemeinen den Schnitt genau in der Medianebene vor, besonders dann, wenn es darauf ankommt, bakteriologische Untersuchungen anszufübren. Die Nasenscheidenwand wird bei einiger Vorsicht dabei halbiert, besonders leicht am kindlichen Schädel. Wie bei Harke wird der Sehnitt möglichst tief zur Nasenwurzel geführt, dann werden die beiden Teile der vorderen Schädelhälfte auseinandergebrochen und anfgeklappt (Textfig. 2), so daß beide Nasenhälften fast in einer horizontalen Ebene liegen und bequem zu- 
gänglich sind. Damit werden meistens auch die beiden Stirnhöhlen eröffnet. Wurde der sagittale Schnitt genau in der Mitte geführt, so bleiben die Nasenhöhlen zunächst von den beiden Hälften der Nasenscheidenwand bedeckt und geschützt und können einzeln unter allen erforderlichen Kautelen aufgemacht wexden (Textfig. 2).

Nach Vollendung der den Nasenhöhlen zugedachten Untersuchung werden nun die Siebbeinzellen jeder Seite dadurch eröffnet, daß fast parallel dem sagittalen Sehnitt oder nach vorn zu etwas medial abweichend ein Sägeschnitt von hinten nach vorn durch die Nasenseitenwand gefïhrt wird, der diese mit der oberen und mittleren Muschel abträgt und die ganze Reihe der Siebbeinzellen überaus übersichtlich eröffnet, so daB Zelle für Zelle besichtigt und gegebenenfalls von jeder einzelnen Zelle Inhalt zur Untersuchung gewonnen werden kann. (Textfig. 3).

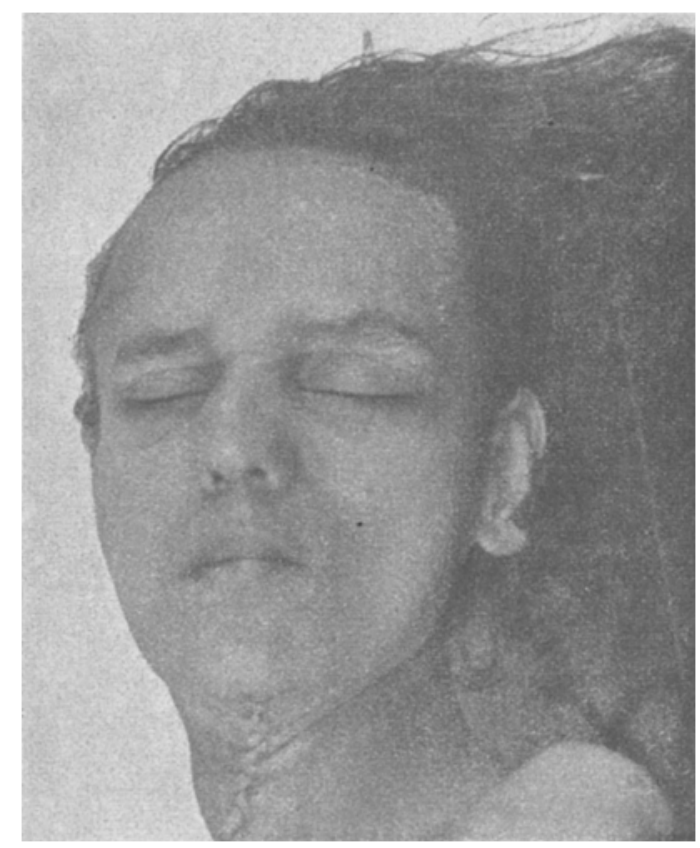

Fig. 5.

Ist dies auch anf der andern Seite geschehen, so werden schlieblich nach Abtragung der noch vorhandenen Nasenmuscheln oder ihrer Reste von der Nasenseitenwand aus die Highmoreshöhlen eröffnet (Textfig. 4).

Da die Gehörorgane bei dieser Methode ganz der durch den frontalen Schnitt geschaffenen hinteren Hälfte des Schädels zufallen, ist es möglich, auch eine Untersuchung des Gehörorganes anzuschließen, sei es durch Herausnahme der Felsenbeinpyramide oder durch Eröffnung des Tegmen tympani.

Eine Verunstaltung der Leiche erfolgt niemals. Nach Vollendung der Untersuchung werden zunächst die beiden Teile der vorderen Schädelhälfte wieder zusammengeklappt, hernach die vordere und hintere Schädelhälfte und darüber die beiden Teile der Kopfschwarte gezogen und genäht (Textfig. 5). 


\section{Literatur.}

Beneke, Zur Technik der Oberkiefer- und Nasenhöhlensektion. Ztlbl. f. a. Path. u. path. Anat. 1896. - Chiari, H., Pathologisch-anatomische Sektionstechnik, 2. Aufl., 1907. Hansemann, D. v., Die Luxation des Schädels als Sektionsmethode. Ztlbl. f. anat. Path. u. path. Anat. 1909. - Harke, Ein neues Verfahren, die Nasen- und Rachenhöhle mit ihren pneumatischen Anhängen am Leichnam ohne Entstellung freizulegen. Virch. Arch. 125. Bd., 1891. Hauser, G., Die Zenkersche Sektionstechnik, 1913. - Klebs, E., zit. nach Nauwerck. Löwe, Über eine neue Sektionsmethode der Nasenhöhlen und der angrenzenden Teile der Orbita, des Fpipharynx und der Basis cranii. Virch. Arch. 163. Bd., 1901. - Loeschcke, Demonstrationen zur Sektionstechnik. Verhdl. d. D. Path. Ges., 17. Tagung, 1914. - Nauwerck, C., Sektionstechnik, 5. Aufl., 1912. - Oberndorfer, Die Lösung der Schädelbasis als Sektionsmethode. Ztlbl. f. anat. Path. u. path. Anat. 1909. - Schalle, Eine neue Sektionsmethode für die Nasen-Rachen- und Gehörorgane. Virch. Arch. 71. Bd., 1877. - Westenhoeffer, Atlas der path.-anat. Sektionstechnik, 1908. 